\title{
NEWTON POLYHEDRA (ALGEBRA AND GEOMETRY)
}

\author{
A.G. KhOvanskiI
}

Amer. Math. Soc. Transl. (2) Vol. 153, 1992

Newton polyhedra establish a relationship between algebraic geometry and the geometry of polyhedra. In this paper we discuss this relationship and its applications in algebra and geometry.

\section{$\S 1$. Computation of Discrete invariants IN TERMS OF NEWTON POLYHEDRA}

In this section we discuss the computation of discrete algebraic invariants in geometric terms and, in particular, the computation of the number of roots of a system of equations in terms of the mixed volumes of polyhedra. An outline of an algebraic proof of the isoperimetric inequality will be given.

1.1. The ideology of general position. Suppose that the outcome of some natural process, such as a physical experiment, is the graph of a function of one variable over a closed interval of finite length. One feels that such a function ought to have only finitely many roots. Can this be proved rigorously? "Classical mathematics" answers this question with an univocal "no": for any given closed subset of a closed interval (such as a Cantor perfect set) there exists an infinitely differentiable function whose set of zeroes is the given set. In the "mathematics of general position", however, the statement can be proved: The space of smooth functions over a closed interval contains a dense open set of functions which have finitely many zeroes, maxima, and minima. A "generic" function is a function from a dense open set. "Non-generic" functions form a hypersurface of codimension one in the space of functions. Various pathological effects, such as the existence of infinitely many zeroes over a closed interval of finite length, may of course occur, but for the overwhelming majority of functions no complications arise. It is natural to expect that a function occurring in some natural context (and not constructed specially as a counterexample) will be a function in general position.

For various classes of objects (functions, mappings, differential equations) one can often single out those in general position, which, first, constitute the overwhelming majority, and, second, behave in a much simpler way than an arbitrary object.

The main task of singularity theory is to identify and investigate objects in general position. To illustrate how fruitful this approach can be, let us compare the following two assertions:

1) There is no algebraic algorithm that enables us to determine, using only the coefficients of a differential equation, whether or not its equilibrium position is stable. 
2) For "generic" differential equations there is an explicit (algebraic) procedure that will tell us whether an equilibrium is stable or not.

Thus, the unsolvability of the stability problem, as declared by "classical mathematics", pertains only to "special" equations, which should never be encountered in real life except, of course, under special circumstances. Had mathematicians unquestioningly accepted the quite convincing theorem that there is no algorithm for the stability problem and failed to investigate the case of general position, we would have remained ignorant of a remarkable criterion for stability which has innumerable applications and may be found in any textbook on differential equations.

1.2. Complex and real cases in the "mathematics of general position". In both complex and real cases, exceptional objects usually form a hypersurface. In the complex case, however, the hypersurface is also complex and its real codimension is two (one complex equation can be considered as two real ones - a complex number is zero if both its real and imaginary parts are zero). A subset of codimension two does not divide the space into parts, and that is why, starting from one generic complex object, one can reach any other without passing through degenerate, nongeneric objects. Discrete invariants remain unchanged in the process - hence the discrete invariants of any two complex generic objects are equal. Not so in the real case: a generic real hypersurface divides the space into parts and even discrete invariants of different generic real objects may be distinct. Here is a simple example: in the space of polynomials of degree $\mathrm{n}$ in one complex variable, the nongeneric polynomials having multiple roots form a complex hypersurface. The number of complex roots of a generic complex polynomial of degree $\mathrm{n}$ does not depend on the choice of the polynomial - it is $n$. On the other hand, the number of real roots of a generic real polynomial depends on the choice of the polynomial (it may be $n,(n-2),(n-4)$, dots ).

1.3. Examples of computation with Newton polyhedra. Newton polyhedra generalize the notion of the degree of a polynomial and playa similar role. The Newton polyhedron of a polynomial of $n$ variables is a polyhedron in the real linear space $\mathbb{R}^{n}$, defined as the convex hull of the points whose coordinates are the exponents of the powers that occur in the polynomial with nonzero coefficients.

FIGURE 1.

Examples. 1. Consider the following polynomial $p$ in $x$ and $y$ :

$$
y^{2}+Q_{3}(X)=y^{2}+a_{0}+a_{1} x+a_{2} x^{2}+a_{3} x^{3} .
$$


The Newton polygon $\Delta(p)$ of this polynomial is shown in Figure 1. The point $(0,2)$ in this figure corresponds to the monomial $y^{2}$, the points $(0,0),(0,1),(0,2)$, and $(0,3)$ to the monomials $a_{0}, a_{1} x, a_{2} x^{2}$, and $a_{3} x^{3}$, respectively.

2. The Newton polyhedron of a generic polynomial of degree $m$ in two variables is the simplex with vertices $(m, 0),(0, m),(0,0)$.

The Newton polyhedron of a generic polynomial of degree $m$ in $n$ variables is the $n$-dimensional simplex $x_{1} \geq 0, \ldots, x_{n} \geq 0, \sum x_{i} \leq m$. Thus we see that Newton polyhedra indeed generalize the notion of degree.

Discrete parameters of the complex variety defined by a generic equation $P(x)=$ 0 with fixed Newton polyhedron $\Delta(P)$ do not depend on the specific choice of the equation; they depend only on $\Delta(P)$.

Example. Consider a generic complex curve $P(x, y)=0$ in the plane, with fixed Newton polyhedron $\Delta$. Its genus is equal to the number of points with integer coordinates in the interior of the Newton polyhedron $\Delta$ [1]. Thus, the curve $y^{2}+Q_{3}(x)=0$, where $Q_{3}(x)$ is a sufficiently generic polynomial of third degree, has genus 1, since its Newton poly- gon contains only one integral point (with coordinates $(1,1)$; see Figure 1). Similarly, the genus g of a generic curve $P_{m}(x, y)$ of degree $m$, is $g=(m-1)(m-2) / 2$, which is just the number of points in the interior of the simplex $x_{1} \geq 0, x_{2} \geq 0, x_{1}+x_{2} \leq m$.

The formula for the genus of a generic curve is well known. We see that computations with Newton polyhedra generalize the classical computations of invariants of algebraic varieties, which are defined by generic equations of fixed degrees, and give answers a geometrical meaning. Here is a multidimensional generalization of the previous example.

Example. The arithmetical genus of the hypersurface defined in $\mathbb{C}^{n}$ by a generic equation $P(x)=0$ with fixed Newton polyhedron is equal to the number of points with integer coordinates in the interior of the New- ton polyhedron [1]. The corresponding classical computation: the arithmetical genus of a general hypersurface of degree $m$ in $n$-dimensional space is $(m-1) \ldots(m-n) n$ !; this number is just the number of points with integer coordinates inside the simplex $x_{1} \geq 0, \ldots, x_{n} \geq 0$, $\sum x_{i} \leq m$.

As a rule, the answers become simpler and more symmetric if one considers solutions of equations not in $\mathbb{C}^{n}$, but in $(\mathbb{C} \backslash 0)^{n}$, that is, the subdomain of $\mathbb{C}^{n}$ obtained by deleting all the coordinate hyperplanes.

Example [1]. The Euler characteristic of the hypersurface defined in $(\mathbb{C} \backslash 0)^{n}$ by a generic equation $P(x)=0$ with fixed Newton polyhedron is equal to the volume of the polyhedron multiplied by $(-1)^{n-1} n$ !. (The Euler characteristic of a hypersurface in $\mathbb{C}^{n}$ is given by a more complicated formula. However, since Euler characteristic is additive, this more complicated formula reduces to the previous formula.)

As simple an object as the volume of the convex hull of a system of points is an extremely complicated function of the coordinates. Such objects, considered in the usual numerical terms, are so cumbersome that no progress would be possible without a knowledge of their geometrical interpretation. 
1.4. Number of roots of $n$ equations in $n$ unknowns. By the Bézout Theorem, the number of nonzero roots of a polynomial $f$ in one variable is equal to the difference between the maximum and minimum degrees of the monomials occurring in the polynomial. The Newton polyhedron of the polynomial $f$ is a closed interval and the above-mentioned difference is the length of this interval. This observation is generalized to the multidimensional case in the following theorems.

Kushnirenko's Theorem [2]. The number of solutions in $(\mathbb{C} \backslash 0)^{n}$ of a generic polynomial system of $n$ equations $P_{1}=\cdots=P_{n}=0$ with identical Newton polyhedra $\Delta\left(P_{1}\right)=\cdots=\Delta\left(P_{n}\right)=\Delta$ is equal to the volume $V(\Delta)$ of the Newton polyhedron multiplied by $n$ !.

Example. As already stated, the Newton polyhedron of a polynomial of degree $m$ in $n$ variables is a simplex $x_{1} \geq 0, \ldots, x_{n} \geq 0, \sum x_{i} \leq m$ (on the assumption that the polynomial contains all monomials of degrees $\leq m$ ). The volume of this simplex is $m^{n} / n$ !. By Kushnirenko's Theorem, the number of roots of a general system of $n$ equations of degree $m$ in $n$ unknowns is $m^{n}$.

This answer agrees with the Bézout Theorem. The Newton polyhedron of a polynomial that does not contain all monomials of degree $\leq m$ may be smaller than a simplex, and the number of solutions according to Kushnirenko's Theorem may be less than the number $m^{n}$ given by the Bézout Theorem. The reason is that, as some monomials do not appear, some points at infinity may turn out to be roots. Since the Bézout Theorem computes the number of roots in the projective space, it counts these "parasitic" roots, while Kushnirenko's Theorem does not.

Bernstein's Theorem $[\mathbf{3}, \mathbf{1}]$. The number of solutions in $(\mathbb{C} \backslash O)^{n}$ of a generic system of $n$ polynomial equations $P_{1}=\cdots=P_{n}=0$ with Newton polyhedra $\Delta_{1}, \ldots, \Delta_{n}$ is equal to the mixed volume $V\left(\Delta_{1}, \ldots, \Delta_{n}\right)$ multiplied by $n !$.

We first define mixed volume.

By the Minkowski sum of two subsets of a linear space we mean the set of all sums of pairs of vectors, one from the first subset and another from the second one. The product of a subset by a number is defined similarly. The Minkowski sum of convex bodies (convex polyhedra, convex polyhedra with vertices at points with integer coordinates) is a convex body (convex polyhedron, convex polyhedron with vertices at points with integer coordinates). The role of Minkowski summation in the theory of Newton polyhedra is due to the following simple proposition: the Newton polyhedron of a product of polynomials is the Minkowski sum of their Newton polyhedra.

Minkowski's Theorem. The volume of a body that is a linear combination with positive coefficients of fixed convex bodies in $\mathbb{R}^{n}$ is a homogeneous polynomial of degree $n$ in the coefficients of the linear combination.

Definition. The mixed volume $V\left(\Delta_{1}, \ldots, \Delta_{n}\right)$ of convex bodies $\Delta_{1}, \ldots, \Delta_{n}$ in $\mathbb{R}^{n}$ is the coefficient of $\lambda_{1} \ldots \lambda_{n}$ in the polynomial $V\left(\lambda_{1} \Delta_{1}+\cdots+\lambda_{n} \Delta_{n}\right)$, divided by $n$ !, where $V(\Delta)$ is the volume of $\Delta$.

The mixed volume of $n$ identical bodies is equal to the volume of any one of them. The mixed volume of $n$ bodies is expressed in terms of usual volumes of their sums in the same way as the product of $n$ numbers is expressed in terms of 
the $n$th powers of their sums. For example, for $n=2$ we have

$$
\begin{gathered}
a b=\frac{1}{2}\left[(a+b)^{2}-a^{2}-b^{2}\right], \\
V\left(\Delta_{1}, \Delta_{2}\right)=\frac{1}{2}\left[V\left(\Delta_{1}+\Delta_{2}\right)-V\left(\Delta_{1}\right)-V\left(\Delta_{2}\right)\right] .
\end{gathered}
$$

Similarly, for $n=3$,

$$
V\left(\Delta_{1}, \Delta_{2} \Delta_{3}\right)=\frac{1}{3 !}\left[V\left(\Delta_{1}+\Delta_{2}+\Delta_{3}\right)-\sum_{i<j} V\left(\Delta_{i}+\Delta_{j}\right)+\sum_{i} V\left(\Delta_{i}\right)\right] .
$$

Example. Let $\Delta_{1}$ be the rectangle $0 \leq x \leq a, 0 \leq y \leq b$, and $\Delta_{2}$ the rectangle $0 \leq x \leq c, 0 \leq y \leq d$. The Minkowski sum $\Delta_{1}+\Delta_{2}$ is the rectangle $0 \leq x \leq a+c$, $0 \leq y \leq b+d$. The mixed volume $V\left(\Delta_{1}, \Delta_{2}\right)$ is $a d+b c$. The number $a d+b c$ is the permanent of the matrix $\left(\begin{array}{ll}a & b \\ c & d\end{array}\right)$ (the definition of the permanent is the same as that of the determinant, except that all summands enter with the plus sign). In the multidimensional case the mixed volume of $n$ parallelepipeds whose sides are parallel to the coordinate axes is also equal to the permanent of the corresponding matrix.

Example. The number of roots of a generic system of polynomial equations in which the $i$ th variable occurs in the $j$ th equation to power at most $a_{i, j}$ is equal to the permanent of the matrix $\left(a_{i, j}\right)$ times $n$ !.

For equations with identical Newton polyhedra, the statements of Kushnirenko's and Bernstein's theorems coincide.

Newton polyhedra can be used to compute not only discrete invariants of hypersurfaces and numbers of solutions of systems of $n$ equations in $n$ unknowns, but also discrete invariants of the varieties of solutions of systems of $k$ equations in $n$ unknowns. Here is a very simple example.

Example [4]. For $0 \leq i<n-k$, the $i$-dimensional homology group of the variety $X$ of solutions of a generic system of $k$ polynomial equations $P_{1}=\cdots=P_{k}=0$ in $(\mathbb{C} \backslash O)^{n}$ is isomorphic to the $i$-dimensional homology group of the space $(\mathbb{C} \backslash O)^{n}$, if the Newton polyhedra $\Delta, \ldots, \Delta^{k}$ of the system have full dimension, i.e., $\operatorname{dim} \Delta_{1}$, = $\cdots=\operatorname{dim} \Delta_{k}=n$. Hence, $\operatorname{dim} H_{i}(X)=\left(\begin{array}{c}n \\ i\end{array}\right)$, for $0 \leq i<n-k$. In particular, if $k<n$, the variety $X$ is connected.

Let us return now to mixed volumes.

1.5. Aleksandrov-Fenchel inequalities. Many geometrical invariants can be expressed in terms of mixed volumes of bodies. For example, the $\varepsilon$-neighborhood of a convex body $\Delta$ is the set $\Delta+\varepsilon K$, where $K$ is the unit ball about the origin. By the Minkowski Theorem, the volume of the $\varepsilon$-neighborhood of a body $\Delta$ is the following polynomial in $\varepsilon$ :

$$
V(\Delta)+n \varepsilon V_{n-1}(\Delta)+\frac{n(n-1)}{2} \varepsilon^{2} V_{n-2}(\Delta)+\ldots,
$$


where

$$
V_{l}(\Delta)=V(\underbrace{\Delta, \ldots, \Delta}_{l \text { times }}, \underbrace{K, \ldots, K}_{n-l \text { times }}) .
$$

On the other hand, it is clear that in the first approximation with respect to $\Delta$ the volume of the $\varepsilon$-neighborhood of $\Delta$ is $V(\Delta)+\varepsilon S(\Delta)$, where $S(\Delta)$ is the surface area of $\Delta$. From this we conclude that the surface area of $\Delta$ is $n V_{n-1}(\Delta)$. (It is not difficult to show that the mixed volume $V_{l}(\Delta)$ coincides, up to a factor depending only on $l$ and $n$ with the mean value of the area of the projection of $\Delta$ onto an $l$-dimensional plane.)

The following remarkable inequality of Aleksandrov-Fenchel for mixed volumes of bodies is a generalization of the isoperimetric inequality

$$
V^{2}\left(K_{1}, K_{2} \ldots, K_{n}\right) \geq V\left(K_{1}, K_{1} \ldots, K_{n}\right) V\left(K_{2}, K_{2}, \ldots, K_{n}\right)
$$

where $K_{1}, \ldots, K_{n}$ are nonempty convex bodies. The following assertion is a simple formal corollary of these inequalities: for any two convex bodies $K_{1}$ and $K_{2}$ and any integer $l, 0<l<n$,

$$
V^{n}(\underbrace{K_{1} \ldots, K_{1}}_{l \text { times }} \underbrace{K_{2}, \ldots, K_{2}}_{n-l \text { times }}) \geq V^{l}\left(K_{1}\right) \cdot V^{n-l}\left(K_{2}\right) .
$$

Applying this assertion to the case when $K_{1}$ is $\Delta, K_{2}$ is the unit ball $K$, and $l=n-1$, we obtain the isoperimetric inequality:

$$
S(\Delta) \geq V^{(n-1) / n}(\Delta) V^{1 / n}(K) .
$$

The isoperimetric inequality furnishes an upper bound for the volume of a body in terms of its surface area. This bound is best possible: for a ball the equality sign holds.

The proof of the Aleksandrov-Fenchel inequalities is far from simple. Three geometrical proofs of these inequalities are known. Two were published by A.D. Aleksandrov in 1936 [5]. One of them is combinatorical (the proof is worked out for polyhedra and then extended to all convex bodies by continuity). The other is analytical; it uses the theory of selfadjoint elliptic operators depending on parameters (the proof is worked out for smooth, strictly convex bodies and then extended to all convex bodies by continuity). Fenchel's geometrical proof also dates to 1936 (it is so complicated that it has never been published in full).

1.6. An algebraic proof of the Aleksandrov-Fenchel inequalities. Thanks to the link created by Newton polyhedra between algebraic geometry and the geometry of polyhedra, algebraic theorems of general type yield considerable information about the geometry of polyhedra. As an example, we will show how the Hodge Index Theorem can be used to obtain a simple proof of the Aleksandrov-Fenchel Theorem. The proof outlined below was found independently in 1979 by Teissier $[6,7]$ and the author $[8,9]$.

We first recall the Hodge Inequality from the algebraic geometry of surfaces [10], which will play a key role in what follows. Let $\Gamma_{1}$ and $\Gamma_{2}$ be two complex curves on a compact connected complex algebraic surface $F$. Let the self-intersection number of one of the curves be positive. The Hodge Inequality states that

$$
\left\langle\Gamma_{1}, \Gamma_{2}\right\rangle^{2} \geq\left\langle\Gamma_{1}, \Gamma_{1}\right\rangle\left\langle\Gamma_{2}, \Gamma_{2}\right\rangle,
$$


where $\left\langle\Gamma_{1}, \Gamma_{2}\right\rangle$ is the intersection number of the curves $\Gamma_{1}$ and $\Gamma_{2},\left\langle\Gamma_{1}, \Gamma_{1}\right\rangle$ and $\left\langle\Gamma_{2}, \Gamma_{2}\right\rangle$ are self-intersection numbers of these curves. (Recall that the self-intersection number of a curve is defined as the intersection number of the curve with its image under a slight deformation.)

The Aleksandrov-Fenchel inequalities will be inferred from the Hodge Inequality and Bernstein's Theorem, as follows.

Consider the noncompact algebraic surface defined in $\mathbb{C}^{n}$ by a generic system of $(n-2)$ polynomial equations $P_{3}=0, \ldots, P_{n}=0$ with Newton polyhedra $\Delta 3, \ldots, \Delta n$ of full dimension $\operatorname{dim} \Delta_{3}=\cdots=\operatorname{dim} \Delta_{n}=n$. Since by assumption the Newton polyhedra are of full dimension, the surface will be connected. Let $A_{1}$ and $A_{2}$ be two curves on this surface, defined by generic polynomial equations $P_{1}=0$ and $P_{2}=0$ with Newton polyhedra $\Delta_{1}$ and $\Delta_{2}$. The number of points of intersection of the curves $A_{1}$ and $A_{2}$ on our noncompact surface is equal to the number of solutions of the system of equations $P_{1}=P_{2}=P_{3}=\cdots=P_{n}=0$. By Bernstein's Theorem, this number is equal to $n ! V\left(\Delta_{1}, \Delta_{2}, \Delta_{3}, \ldots, \Delta_{n}\right)$. Together with the curve $A_{1}$, let us consider a slightly deformed curve $A_{1}^{\prime}$, defined by an equation $P_{1}^{\prime}=0$ that contains the same monomials as the equation $P_{1}=0$ but with slightly different coefficients (in particular, the polynomials $P_{1}$ and $P_{1}^{\prime}$ have identical Newton polyhedra). Again by Bernstein's Theorem, the number of points of intersection of the curves $A_{1}$ and $A_{1}^{\prime}$ is $n ! V\left(\Delta_{1}, \Delta_{1}, \Delta_{3}, \ldots, \Delta_{n}\right)$. Similarly, we construct a curve $A_{2}^{\prime}$ that intersects the curve $A_{2}$ at $n ! V\left(\Delta_{2}, \Delta 2, \Delta_{3}, \ldots, \Delta_{n}\right)$ points.

The next step is compactification of the noncompact surface $P_{3}=\cdots=P_{n}=0$.

There is a special compactification $F$ of this surface under which the closures $\Gamma_{1}, \Gamma_{1}^{\prime}, \Gamma_{2}$ and $\Gamma_{2}^{\prime}$ of the noncompact curves $A_{1}, A_{1}^{\prime}, A_{2}$, and $A_{2}^{\prime}$ do not intersect "at infinity". Such compactifications play an important technical role in the theory of Newton polyhedra. Some idea of such compactifications will be given in the next section. Since the curves $\Gamma_{1}=\bar{A}_{1}$ and $\Gamma_{1}^{\prime} 1=\bar{A}_{1}^{\prime}$ have no points of intersection "at infinity", it follows that $\Gamma_{1} \cap \Gamma_{1}^{\prime}=A_{1} \cap A_{1}^{\prime}$; similar equalities hold for the other pairs of curves. Hence the intersection and self-intersection numbers of the curves $\Gamma_{1}$ and $\Gamma_{2}$ are determined by the formulas

$$
\begin{aligned}
& \left\langle\Gamma_{1}, \Gamma_{2}\right\rangle=n ! V\left(\Delta_{1}, \Delta_{2}, \Delta_{3}, \ldots, \Delta_{n}\right), \\
& \left\langle\Gamma_{1}, \Gamma_{1}\right\rangle=n ! V\left(\Delta_{1}, \Delta_{1}, \Delta_{3}, \ldots, \Delta_{n}\right), \\
& \left\langle\Gamma_{2}, \Gamma_{2}\right\rangle=n ! V\left(\Delta_{2}, \Delta_{2}, \Delta_{3}, \ldots, \Delta_{n}\right) .
\end{aligned}
$$

Substituting these formulas into Hodge's Inequality, we obtain the AleksandrovFenchel inequality for the polyhedra $\Delta_{1}, \Delta_{2}, \Delta_{3}, \ldots, \Delta_{n}$ :

$$
V^{2}\left(\Delta_{1}, \Delta_{2}, \Delta_{3}, \ldots, \Delta_{n}\right) \geq V\left(\Delta_{1}, \Delta_{1}, \Delta_{3}, \ldots, \Delta_{n}\right) V\left(\Delta_{2}, \Delta_{2}, \Delta_{3}, \ldots, \Delta_{n}\right)
$$

It follows that the Aleksandrov-Fenchel inequality holds for polyhedra of full dimension with vertices at rational points (by a scale change one can place the vertices at points with integer coefficients). To complete the proof, it remains to use the continuity of mixed volume.

1.7. Minding's method. We conclude this section with a brief discussion of Bernstein's Theorem. To be precise, we will explain to the reader why it is possible to compute the number of roots of a system of two polynomial equations $f(x, y)=$ 
$g(x, y)=0$ using the Newton polygons of polynomials $f$ and $g$. The method that will be discussed below is due to Ferdinand Gottlieb Minding (1806-1865). Minding expressed the answer in terms of the geometrical characteristics of polygons [11]. However, he was not famil- iar with the notion of mixed volume - when his paper was published (1841), Minkowski had not yet been born; he was therefore unable to extend his the- orem to the multi-dimensional case, and the generalization was accomplished only in 1975 by D.N. Bernstein. We now outline Minding's method. Minding eliminates the variable y from the system $f(x, y)=g(x, y)=0$. To that end, he considers the multi-valued function $y(x)$ defined by the equation $f(x, y)=0$ and substitutes it into the second equation. The number of branches of the algebraic function $y(x)$ is equal to the degree of the polynomial $f$ with respect to the variable $y$. Suppose that the degree is $k$, and let $y_{1}(x), \ldots, y_{k}(x)$ be the different branches of the function $y(x)$. The product of all $k$ branches $g\left(x, y_{i}(x)\right)$ of the multi-valued function $g(x, y(x))$ gives a function $p(x)=\prod_{1 \leq j \leq k} g\left(x, y_{i}(x)\right)$; this function is, however, single-valued. It is clear that the zeroes of the function $p$ correspond to the roots of the original system. Since $p$ is a single-valued algebraic function, it is rational. Moreover, if the curve $f(x, y)=0$ has no vertical asymptotes, then $p(x)$ will never become infinite for finite $x$, so it is a polynomial. Therefore, to determine the number of roots of the system we must find the number of roots of the polynomial $p(x)$, which is simply its degree. To determine the degree of the polynomial $p$, Minding suggests the following method. First compute the leading terms of the expansions of the branches $y_{i}(x)$ of $y(x)$ in fractional powers of $x$ (the so-called Puiseux series) as $x \rightarrow \infty$. Then, substituting the leading terms of the expansions of $y_{i}(x)$ into $g(x, y)$, compute the leading terms of the expansions of the branches $g\left(x, y_{i}(x)\right)$ and determine their (fractional) degrees. The required degree of the polynomial $p$ is equal to the sum of the degrees of the branches $g\left(x, y_{i}(x)\right)$ (this sum is always an integer).

Now Newton invented "his" polygons for the very purpose of determining the leading terms of expansions of algebraic functions in Puiseux series. Minding uses the Newton polygon of the polynomial $f(x, y)$ to determine the leading terms in the expansions of branches $y_{i}(x)$. He points out that in general position, when

the coefficients of polynomials $f$ and $g$ are not bound by special relationships, the leading terms of the branches $y_{i}(x)$ do not cancel out after being substituted into $g(x, y)$. In addition, the degree of any branch $g\left(x, y_{i}(x)\right)$ depends only on that of $y_{i}(x)$ and on the Newton polygon of the polynomial $g(x, y)$. It is noteworthy that Minding's algorithm for computing the number of solutions of the system $f(x, y)=g(x, y)=0$ as the degree of the polynomial $p=\prod g\left(x, y_{i}(x)\right)$, obtained by expanding the branches $y_{i}(x)$ in Puiseux series, also works in the degenerate case. In the case of degeneration, however, leading terms may cancel out, so that the number of roots and the degree of the polynomial $p$ can decrease.

\section{TORIC VARIETIES AND THE COMBINATORICS OF POLYHEDRA}

In this section we shall give an intuitive idea of toric varieties and describe an application of the algebraic geometry of toric varieties to the combinatorics of polyhedra.

2.1. The notion of toric variety. It is well known that generic curves of degree $m$ are far more easily and conveniently studied not in the plane $\mathbb{C}^{2}$, but in the projective plane $\mathbb{C} I P^{2}$. This is because, first, the projective plane is compact and, 
second, the generic curve of degree $m$ in the projective plane is smooth (compactification introduces no singularities "at infinity").

\section{FIGURE 2.}

Figure 2 is a schematic representation of the projective plane, showing the $x$ and $y$ axes and the line at infinity.

Now this picture clearly looks like the Newton polygon of a generic polynomial of degree $m$. Moreover, the monomials of the highest degree $m$ on the side $x_{1}+x_{2}=m$, $x_{1} \geq 0, x_{2} \geq 0$ of the Newton polygon play the main role when one approaches the line at infinity. The monomials on the side $x_{1}=0, x_{2} \geq 0, x_{1}+x_{2} \leq m$ of the Newton polygon dominate near the $x$ axis, while monomials on the side $x_{1} \geq 0$, $x_{2}=0, x_{1}+x_{2} \leq m$ are dominant near the $y$ axis.

In the same way, a curve defined by a generic equation with a fixed Newton polygon $\Delta$ is more easily and conveniently considered in a special compactification of the space $(\mathbb{C} \backslash 0)^{2}$ based on the polygon $\Delta$. To each side of the polygon $\Delta$ corresponds a deleted straight line $\mathbb{C} \backslash 0$, which is added to $(\mathbb{C} \backslash 0)^{2}$ during the compactification, and to each vertex of the polygon $\Delta$ corresponds an added point. The monomials on anyone side of the Newton polygon become dominant when one approaches the corresponding deleted line, and the monomials at the vertices of the Newton polygon dominate when one approaches the corresponding points.

In the multi-dimensional case we can also construct compactifications of the space $(\mathbb{C} \backslash O)^{n}$ based on Newton polyhedra [12]. They play the same role as the projective compactification for general varieties of fixed degrees. All these compactifications are what is known as toric varieties [13].

The space $(\mathbb{C} \backslash O)^{n}$ is an algebraic group with respect to coordinatewise multiplication of vectors, known as an $n$-dimensional torus. A connected $n$-dimensional algebraic variety (in general, singular) on which an $n$-dimensional torus acts algebraically and has one orbit isomorphic to an $n$-dimensional torus, is called a toric variety. Under the action of a torus, a toric variety is broken up into a finite number of orbits isomorphic to tori of different dimensions. To every Newton polyhedron $\Delta$ we can associate a compact projective toric variety in such a way that every $k$-dimensional face of $\Delta$ corresponds to a complex $k$-dimensional orbit in the toric variety. If one face of the polyhedron is contained in the closure of another face, then the orbit corresponding to the first face is contained in the closure of the orbit corresponding to the second one. We will not present the construction of the toric variety associated with a Newton polyhedron $\Delta$, but restrict ourselves to the following example. 
Example. The torus $(\mathbb{C} \backslash 0)^{2}$ acts on the projective plane $\mathbb{C} P^{2}$ by the following formula: an element $\left(u_{1}, u_{2}\right) \in(\mathbb{C} \backslash 0)^{2}$ takes the point $z_{1}: z_{2}: z_{3}$ into the point $u_{1} z_{1}: u_{2} z_{2}: z_{3}$. With this action, $\mathbb{C} P^{2}$ becomes a two-dimensional toric variety. This variety corresponds to the Newton polygon of a generic polynomial of any fixed degree $m>0$. The sides of the polygon along the lines $x_{1}=0, x_{2}=0$, and $x_{1}+x_{2}=m$ correspond to the one- dimensional orbits $z_{1}=0, z_{2} z_{3} \neq 0 ; z_{2}=0$, $z_{1} z_{3} \neq 0$; and $z_{3}=0, z_{1} z_{2} \neq 0$. The vertices $(0,0),(m, 0)$, and $(0, m)$ correspond to the zero-dimensional orbits $(0: 0: 1),(1: 0: 0)$, and $(0: 1: 0)$.

2.2. Simple polyhedra. We now turn to the combinatorics of polyhedra. A bounded polyhedron is said to be simple if it is an intersection of half- spaces in general position. An $n$-dimensional simple polyhedron has the same structure near each vertex as the positive orthant in $\mathbb{R}^{n}$ near the origin. In particular, each vertex of a simple $n$-dimensional polyhedron is incident with exactly $n$ edges, and any $k$ of these edges belong to one $k$-dimensional face containing the vertex.

Remark. In "classical mathematics" a polyhedron can be defined either as the intersection of a finite number of subspaces or as the convex hull of a finite set of points. A priori, these two definitions determine dual objects, and in the mathematics of "general position" they are indeed distinct. As envisaged by the first definition, a generic polyhedron is a simple polyhedron. From the point of view of the second definition, it is a polyhedron all of whose proper faces are simplices. However, the theory of simple polyhedra differs only slightly from the theory of polyhedra with simplicial faces: polyhedra that are dual to simple polyhedra have simplicial faces, and vice versa.

The $F$-polynomial of a simple $n$-dimensional polyhedron is defined as the generating polynomial of the sequence formed by the numbers of faces of different dimensions in the polyhedron, i.e., $F(t)=\sum F_{k} t^{k}$, where $F_{k}$ is the number of $k$-dimensional faces of the polyhedron.

By no means all polynomials are $F$-polynomials of simple polyhedra. Conditions for a polynomial to have this property were found by McMullen [14]. The necessity of these conditions was proved by Stanley [15], their sufficiency by Billera and Lee [16]. To formulate the conditions we need the notion of the $H$-polynomial of a polyhedron. To each $F$-polynomial we associate an $H$-polynomial defined by the formula $H(t)=F(t-1)$; i.e., if $\mathrm{F}(t)=\sum F_{k} t^{k}$, then $H(t)=\sum H_{k} t^{k}$, where $H_{k}=\sum(-1)^{m-k}\left(\begin{array}{c}m \\ k\end{array}\right) F_{k}$.

We now present McMullen's conditions. For any simple $n$-dimensional polyhedron:

(1) the $H$-polynomial is reflexive, i.e., $H_{k}=H_{n-k}$;

(2) coefficients of an $H$-polynomial increase to the middle, i.e., $H_{0} \leq H_{1} \leq \cdots \leq$ $H_{[n / 2]}$, and the coefficients $H_{0}$ and $H_{n}$ are 1 ;

(3) for any $i$ such that $1 \leq i<[n / 2]$, the difference $H_{i+1}-H_{i}$ satisfies an estimate that depends on the difference $H_{i}-H_{i-1}$; more precisely, $H_{i+1}-H_{i} \leq Q^{i}\left(H_{i}-H_{i-}\right)$. Here $Q^{i}$ is a special function of integers (for the definition of the function $Q^{i}$, see [14]). We shall need this function in subsection 2.3 .

The statement about the reflexivity of the $H$-polynomial is also known as the Dehn-Sommerville Theorem. A special case of that theorem for $k=0$ coincides with the Euler Theorem for polyhedra: $\sum_{i=0}^{n}(-1)^{i} F_{i}=1$. Indeed, the number $H_{0}$ is equal to $\sum_{i=0}^{n}(-l)^{i} F_{i}=1$, while $H_{n}$ is equal to $F_{n}$, which is equal to one since an $n$-dimensional polyhedron has exactly one face in the highest dimension. The 
Dehn-Sommerville Theorem shows that the numbers $F_{i}$ for a simple $n$-dimensional polyhedron obey $[(n+1) / 2]$ linear relations. In particular, if we consider simple 3 -dimensional polyhedra, we have, in addition to the Euler relation, the obvious relation $2 E=3 V$ (where $E$ is the number of edges and $V$ the number of vertices).

The increase of the first half of the coefficients of an $\mathrm{H}$-polynomial is far from trivial; there is still no elementary proof of this fact. The only known proof is based on the theory of toric varieties and algebraic geometry. We shall discuss this proof below. The increase of the first half of the coefficients of an $H$-polynomial implies the following

Corollary. All coefficients $H_{0}, \ldots, H_{n}$ of an $H$-polynomial are positive.

We are now going to prove this corollary and the Dehn-Sommerville Theorem using an elementary argument - a remarkable analogue of Morse theory in linear programming.

Let us say that a linear function on a simple polyhedron is generic if its restriction to any edge is not constant. We say that a generic linear function on a simple polyhedron has index $i$ at a vertex if it decreases along exactly $i$ edges emanating from the vertex (and increases along the remaining $n-i$ edges).

Theorem [17]. The number $h(i)$ of vertices of index $i$ for any generic linear function on a simple polyhedron is exactly $H_{i}$, the $i$ th coefficient of the $H$-polynomial of the polyhedron.

This theorem generalizes the obvious fact that a generic linear function on a simple polyhedron (regardless of the choice of the function) has exactly one maximum (i.e., $h_{n}=1$ ) and one minimum (i.e., $h_{o}=1$ ). Let us proceed to the proof.

It will suffice to show that if $F_{k}$ is the number of $k$-dimensional faces of a simple polyhedron, then $F_{k}=\sum\left(\begin{array}{l}i \\ k\end{array}\right) h(i), k=0, \ldots, n$. Consider the map that assigns to each $k$-dimensional face of the polyhedron the vertex at which the linear function assumes its maximum on that face. Under this map, any vertex of index $i$ will be assigned to exactly $\left(\begin{array}{l}i \\ k\end{array}\right) k$-dimensional faces. Indeed, for any point on a $k$ dimensional face at which the function has a maximum, it decreases along the $k$ edges from the point that lie on the face. Conversely, to any set of $k$ edges from the same vertex, along which the function decreases, there corresponds a $k$ dimensional face, namely the face for which this vertex is a maximum point; in a simple polyhedron, every set of $k$ edges radiating from one vertex spans a $k$ dimensional face. Now summing the number of inverse images of our mapping over all vertices, we obtain the required formula.

Corollary 1. $H_{i}=H_{n-i}$.

Indeed, a vertex of index $i$ for a function $L$ has index $(n-i)$ for the function $-L$. Hence the number $h(i)$ for the function $L$ is precisely the number $h(n-i)$ for the function $-L$.

Corollary 2. For a simple n-dimensional polyhedron the numbers $H_{0}, H_{1}, \ldots, H_{n}$ are strictly positive.

Indeed, for any vertex of a polyhedron and any number $i=0,1, \ldots, n$, there exists a generic linear function that has index $i$ at that vertex. 
2.3. Simple polyhedra and quasismooth toric varieties. As already stated, for every Newton polyhedron one can associate a toric variety. If the Newton polyhedron is simple, then the variety is quasi smooth, i.e., it is isomorphic near each point to a domain in the space $\mathbb{C}^{n}$ modulo the action of a finite group. The cohomology theory of quasismooth varieties is quite similar to the cohomology theory of smooth varieties. On quasismooth varieties one can consider differential forms - in the local charts these are forms in domains of $\mathbb{C}^{n}$ that are invariant under the action of the appropriate groups. On quasismooth varieties, just as on smooth ones, the cohomology carries a (pure) Hodge structure, and one can define numbers $h^{p, q}$ as dimensions of the Hodge subspaces in the $(p+q)$-dimensional cohomology. Just as for smooth varieties, there is the Poincare duality for quasi smooth varieties and we have equalities $h^{p, q}=h^{n-p, n-q}, h^{p, q}=h^{q, p}$. In particular, $h^{i, i}=h^{n-i, n-i}$. In addition, the Strong Lefschetz Theorem holds for such varieties. In particular, this theorem states that, for $n$-dimensional quasismooth varieties, $h^{0,0} \leq h^{1,1} \leq \cdots \leq h^{k, k}$, where $k=[n / 2]$.

We have the following theorem: the number $h^{i, i}$ for the toric variety associated with a simple Newton polyhedron $\Delta$ coincides with the $i$ th coefficient $H_{i}$ of the $H$-polynomial of the polyhedron; all the other numbers $h^{p, q}, p \neq q$, are equal to zero. It is now evident that the symmetry of the coefficients of the $H$-polynomial of a simple polyhedron is a consequence of Poincare duality, while the increase of the first half of the coefficients of the $H$-polynomial of a simple polyhedron is a corollary of the Strong Lefschetz Theorem. In addition, for toric varieties the cohomology classes of Hodge degree $(i, i)$ in the cohomology ring are generated by the cohomology classes of Hodge degree $(1,1)$. In the geometry of polyhedra this is due to the fact that every proper face of a polyhedron is an intersection of proper faces of higher dimensions. Faces of dimension $n-i$ correspond to algebraic subva- rieties of the toric variety of complex dimension $n-i$. The group of cycles spanned by these subvarieties is dual to the Hodge subspace in the cohomology of degree $(i, i)$, and an intersection of faces corresponds to intersection of cycles and multiplication of dual cohomology classes.

For any $n$-dimensional quasismooth variety, whose cohomology of Hodge degree $(i, i)$ is generated by its cohomology of Hodge degree $(1,1)$, we have the inequality $\left(h^{i+1, i+1}-h^{i, i}\right) \leq Q^{i}\left(h^{i, i}-h^{i-1, i-1}\right)$, where $Q^{i}$ is the function appearing in McMullen's conditions and $0<i<[n / 2]$. Thus, the restriction on the rate of increase of the numbers $H_{i}$ for simple polyhedra is also a consequence of toroidal geometry.

The linear programming theorem proved above was also suggested by toroidal geometry. The point is that there exists a special mapping onto a Newtonian polyhedron, called the moment map of the toric variety associated with the polyhedron [17]. Under this map, orbits of complex dimension i go into $i$-dimensional faces of the polyhedron. The superposition of a generic linear function on a Newton polyhedron and the moment map determines a Morse function on the toric variety. The critical points of this function are 0-dimensional orbits. The Morse index of a critical point is twice the index of the linear function at the corresponding vertex. Indeed, the function decreases on a $2 i$-dimensional real variety containing the critical point and increases on a $2(n-i)$-dimensional real variety containing it. Here i is the index of the vertex, and the varieties of decrease and increase are the closures of the orbits corresponding to the $i$-dimensional and $(n-i)$-dimensional faces on which the function decreases and increases. It is obvious that if a Morse function on a variety has only critical points of even index, then the number of points of a fixed 
index $2 i$ does not depend on the choice of the Morse function and is equal to the $2 i$-dimensional Betti number of the variety. That is why toric geometry explains the linear programming theorem proved above. In addition, the above proof of the Dehn-Sommerville Theorem imitates the proof of Poincare duality, which is based on Morse theory and compares the cell complexes of the variety corresponding to Morse functions $f$ and $-f$.

2.4. Combinatorics of polyhedra and discrete groups in Lobachevsky spaces. We now return to the geometry of simple polyhedra.

Nikulin's theorem [18]. The average number of l-dimensional faces on $f$ a $k$ dimensional face of a simple $n$-dimensional polyhedron, $0 \leq l<k \leq(n+1) / 2$, is bounded from above by a certain function of $l, k$, and $n$, which can be written down explicitly. If $n$ tends to infinity, the function tends to the number of l-dimensional faces of a $k$-dimensional cube.

Using Niku1in's Theorem, Vinberg [19] showed that in the Lobachevsky space of dimension geq32 there are no discrete groups generated by reflections with a compact fundamental polyhedron. It is easy to see that the fundamental polyhedron of such a group is simple - this is why Nikulin's Theorem is applicable.

Nikulin proved his theorem using only the Dehn-Sommerville Theorem and the fact that the coefficients of the $H$-polynomial are positive. We have presented above very simple proofs of these properties based on linear programming. These simple proofs have enabled us to prove the following generalization of Nikulin's Theorem.

Theorem [17]. The bound in Nikulin's Theorem is valid not only for simple polyhedra, but also for edge simple polyhedra. (An n-dimensional polyhedron is said to be edge simple ifeach of its edges meets exactly $(n-1)$ faces of higher dimension.)

Using this bound, we can prove the following

Theorem [17, 20]. In a Lobachevsky space of dimension $>995$ there are no discrete groups generated by reflections with a fundamental polyhedron of finite volume.

A fundamental polyhedron of finite volume is edge simple. That is just why the above bound is applicable. The bound on the mean number of 1-dimensional faces on a $k$-dimensional face of an edge simple polyhedron has recently found an unexpected application in the algebraic geometry of surfaces.

2.5. Sections of simple polyhedra. To end this paper, we shall discuss yet another theorem whose proof is based on programming. If estimates the number of faces in any dimension of a generic $l$-dimensional section of a simple $n$-dimensional polyhedron with fixed numbers $F_{0}, \ldots, F_{n}$ of faces of different dimensions. Consider a simple $(n-l)$-dimensional polyhedron, the number $f_{k}$ of whose $k$-dimensional faces, $k \geq(n-l) / 2$, is equal to the number of $(k+l)$-dimensional faces of the initial polyhedron, $f_{k}=F_{k+l}$, and the number of faces of lower dimensions is found by the Dehn-Sommerville Theorem.

Theorem [14]. The number of faces in any dimension of a generic l-dimensional section does not exceed the number of faces in the same dimension of the polyhedron defined above.

Of course, the theorem is obvious for faces of dimensions $\geq(n-l) / 2$. For faces of lower dimensions, however, it gives a nontrivial bound. 
Examples. 1. A plane that does not pass through the vertices of a simple 3dimensional polyhedron with $E$ edges can cut at most $E / 3+2$ edges. This bound is sharp. By tilting the middle section of a prism with $3 k$ edges we can make it cut the upper and lower bases, while still cutting all the lateral faces. Then the resulting section will cut exactly $k+2$ edges.

2. Consider a polyhedron with the combinatorial structure of an $n$-dimensional cube. If we let $n \rightarrow \infty$, a hypersurface can asymptotically cut at most a fraction $(2 \pi)^{-1 / 2} \int_{-c}^{+c} \exp \left(-x^{2} / 2\right) d x$ of the $k$-dimensional edges, where $k \sim c \sqrt{n}$. This bound is sharp.

3. What is the maximum possible number of $k$-dimensional faces in a simple $l$-dimensional polyhedron which has $p$ faces of the highest dimension? The answer to this question is given by the famous Upper-Bound Theorem, proved in 1970 by McMullen [21]. Every simple $l$-dimensional polyhedron with $p$ faces of the highest dimension can be considered as a generic $i$-dimensional section of a $(p-1)$ dimensional simplex. Application of our theorem in this case yields the UpperBound Theorem.

\section{REFERENCES}

1. A. G. Khovanskii, Newton polyhedra and the genus offull intersections, Funktsional. Anal. i Prilozhen. 12 (1978), no. 1, 51-61; English transl. in Functional Anal. Appl. 12 (1978), no. 1.

2. A. G. Kushnirenko, The Newton polyhedron and the number of solutions of a system of $k$ equations in $k$ unknowns, Uspekhi Mat. Nauk 30 (1975), no. 2, 266-267 (Russian).

3. D. N. Bernstein, The number of roots of a system of equations, Funktsional. Anal. i Prilozhen. 9 (1975), no. 3, 1-4; English transl. in Functional Anal. Appl. 9 (1975), no. 3.

4. V. I. Danilov and A. G. Khovanskii, Newton polyhedra and algorithms for computing HodgeDeligne numbers, Izv. Akad. Nauk SSSR Ser. Mat. 50 (1986), no. 5, 925-945; English transl. in Math. USSR-Izv. 29 (1987), no. 2.

5. A. D. Aleksandrov, Towards a theory of mixed volumes of convex bodies, I, Mat. Sb. 2 (1937), no. 5, 947-972 (Russian); II, 2 (1937), no. 6, 1205-1238; III, 3 (1938), no. I, 27-46; IV, 3 (1938), no. 2, 227-251.

6. B. Teissier, Du theoreme de I 'index de Hodge aux inegalites isoperimetriques, C. R. Acad. Sci. Paris Ser. AB 288 (1979), no. 4, A287-A289.

7. B. Teissier, Varietes toriques etpolytopes, Sem. Bourbaki, , no. 555, Lecture Notes in Math., vol. 901, Springer-Verlag, Berlin and New York, 1981, pp. 71-84.

8. A. G. Khovanskii, Geometry of convex polyhedra and algebraic geometry, Uspekhi Mat. Nauk 34 (1979), no. 4, 160-161. (Russian).

9. A. G. Khovanskii, Algebra and mixed volumes, A Series of Comprehensive Studies in Mathematics. Geometry (Y. D. Burago and V. A. Zalgaller, eds.), vol. 285, Springer-Verlag, Berlin and New York, pp. 182-207.

10. D. Mumford, Lectures on curves on an algebraic surface, Princeton Univ. Press, Princeton, N. J., 1966.

11. F. Minding, Uber die Bestimmung des Grades ein durch Elimination hervorgehenden Gleichung, J. Reine Angew. Math. 22 (1841), 178-183; J. Math. Pures Appl., Ser. I 6 (1841), $412-418$.

12. A. G. Khovanskii, Newton polyhedra and toral varieties, Funktsional. Anal. i Prilozhen. 11 (1977), no. 4, 56-64; English transl. in Functional Anal. Appl. 11 (1977), no. 4.

13. G. Kempf, F. Knudsen, D. Mumford, and B. Saint-Donat, NToroidal embeddings, I, Lecture Notes in Math., vol. 339, Springer-Verlag, Berlin and New York, 1973.

14. P. McMullen, The number offaces of simplicial polytopes, Israel J. Math. 9 (1971), 559-570.

15. R. Stanley, The number of faces of a simplicial convex polytope, Adv. in Math. 35 (1980), no. 3, 236-238.

16. L. J. Billera and C. W. Lee, Sufficiency of McMullen 's conditions for f-vectors of simplicial polytopes, Bull. Amer. Math. Soc. 16 (1980), no. 2, 181-185. 
17. A. G. Khovanskii, Hyperplane sections of polyhedra, toric varieties and discrete groups in Lobachevsky space, Funktsional. Anal. i Prilozhen. 20 (1986), no. 1, 50-61; English transl. in Functional Anal. Appl. 20 (1986), no. 1.

18. V. V. Nikulin, On arithmetical groups generated by reflections in Lobachevsky spaces, Izv. Akad. Nauk SSSR Ser. Mat. 44 (1980), no. 5, 637-649,719-720; English transl. in Math. USSR-Izv. 16 (1981), no. 3.

19. E. B. Vinberg, The absence of crystallographic groups of reflections in Lobachevsky spaces of large dimension, Funktsional. Anal. i Prilozhen. 15 (1981), no. 2, 67-68; English transl. in Functional Anal. Appl. 15 (1981), no. 2.

20. M. N. Prokhorov, The absence of discrete groups of reflections with a noncompact fundamental polyhedron of finite volume in Lobachevsky spaces of large dimension, Izv. Akad. Nauk SSSR Ser. Mat. 50 (1986), no. 2, 320-332; English transl. in Math. USSR-Izv. 28 (1987), no. 2.

21. P. McMullen, The maximum number of faces of convex polytopes, Mathematika 17 (1970), $179-184$.

Translated by A. BOCHMAN 